\title{
REVIEW
}

\section{Hypoxia-inducible factors and the prevention of acute organ injury}

\author{
Samuel N Heyman*, Seymour Rosen², Christian Rosenberger ${ }^{3}$ \\ This article is one of eleven reviews selected from the Annual Update in Intensive Care and Emergency Medicine 2011 (Springer Verlag) and \\ co-published as a series in Critical Care. Other articles in the series can be found online at http://ccforum.com/series/annual. Further \\ information about the Annual Update in Intensive Care and Emergency Medicine is available from http://www.springer.com/series/8901
}

\section{Introduction}

Hypoxic preconditioning has long been considered as organ-protective, and its clinical usage has been suggested in elective procedures, such as coronary surgery and organ transplantation. Although the mechanisms have not been clearly elucidated, it has been postulated that changes in cell-membrane composition and upregulation of various cellular protective mechanisms are responsible for a better tolerance of acute injury. Remote preconditioning (i.e., hypoxic stress in one organ conferring resistance to acute hypoxia in other organs) suggests organ cross-talk, perhaps mediated by cytokines and the immune system.

Increased expression of heme-oxygenase (HO)-1, heatshock proteins (HSP), growth factors such as vascular endothelial factor (VEGF), and erythropoietin (EPO) are among the numerous adaptive responses to sublethal injury that are believed to participate in tissue tolerance during subsequent stress. EPO, for instance, is a ubiquitous pleiotropic survival and growth factor that attenuates experimental acute injury in various organ systems, including neuronal, retinal, cardiac, renal, and hepatic tissues. Its clinical efficacy, though suggested in critically ill patients, is yet to be defined [1].

The expression of these protective mediators and many others is regulated by hypoxia-sensing mechanisms through the induction and stabilization of so called hypoxia-inducible factors (HIF) [2]. In this chapter, we will outline the control and action of HIF as key regulators of hypoxic adaptive response, and particularly examine HIF expression during hypoxic stress. We shall discuss recently developed measures that enable HIF

*Correspondence: heyman@cc.huji.ac.il

'Department of Medicine, Hadassah Hosptial, Mt. Scopus, PO Box 24035, 91240 Jerusalem, Israel

Full list of author information is available at the end of the article

(c) 2011 Springer-Verlag Berlin Heidelberg.

This work is subject to copyright. All rights are reserved, whether the whole or part of the material is concerned, specifically the rights of translation, reprinting, reuse of illustrations, recitation, broadcasting, reproduction on microfilm or in any other way, and storage in data banks. Duplication of this publication or parts thereof is permitted only under the provisions of the German Copyright Law of September 9 , 1965 , in its current version, and permission for use must always be obtained from Springer-Verlag. Violations are liable for prosecution under the German Copyright Law. signal modification and describe their potential use in conferring tissue tolerance during incipient organ injury.

\section{HIF regulation and action}

HIFs are heterodimers (Fig. 1), composed of a constitutive $\beta$-subunit (HIF- $\beta$ ) and one of three different oxygendependent and transcriptionally active $\alpha$-subunits, among which HIF- $1 \alpha$ and $-2 \alpha$ are acknowledged as promotors of hypoxia adaptation, whereas the role of HIF- $3 \alpha$ remains unclear. Under normoxia, HIF- $\alpha$ subunits are constantly produced, but not allowed to accumulate, since they are rapidly hydroxylated by oxygen-dependent HIF prolyl-4hydroxylase domain enzymes (PHD), subsequently captured by the ubiquitin ligase Von-Hippel-Lindau protein (VHL), and degraded by the proteasome. Under oxygen deficiency, PHD activity is reduced, HIF- $\alpha$ accumulates within the cytosol, $\alpha \beta$-dimers are formed, translocate into the nucleus, and bind to hypoxia response elements (HREs) in the promoter enhancer region of genes, which are subsequently transactivated [2-4].

The biological effects of the more than 100 acknowledged HIF target genes are multiple, and include key steps in cell metabolism and survival. Many of the HIFtarget genes constitute a reasonable adaptation to hypoxia, such as erythropoiesis (EPO), increased glucose uptake (glucose transporter-1), switch of metabolism to glycolysis (several key enzymes of glycolysis), increased lactate utilization (lactate dehydrogenase), angiogenesis (VEGF), vasodilation (inducible nitric oxide synthase [iNOS]), removal of protons (carbonic anhydrase 9), and scavenging of free radicals (HO-1) [2-4].

\section{Biological and rherapeutic modes of HIF activation}

Every cell type has the potential to upregulate HIF, principally by the inhibition of PHD, under conditions when cellular oxygen demand exceeds oxygen supply,

\section{Bïomed Central}




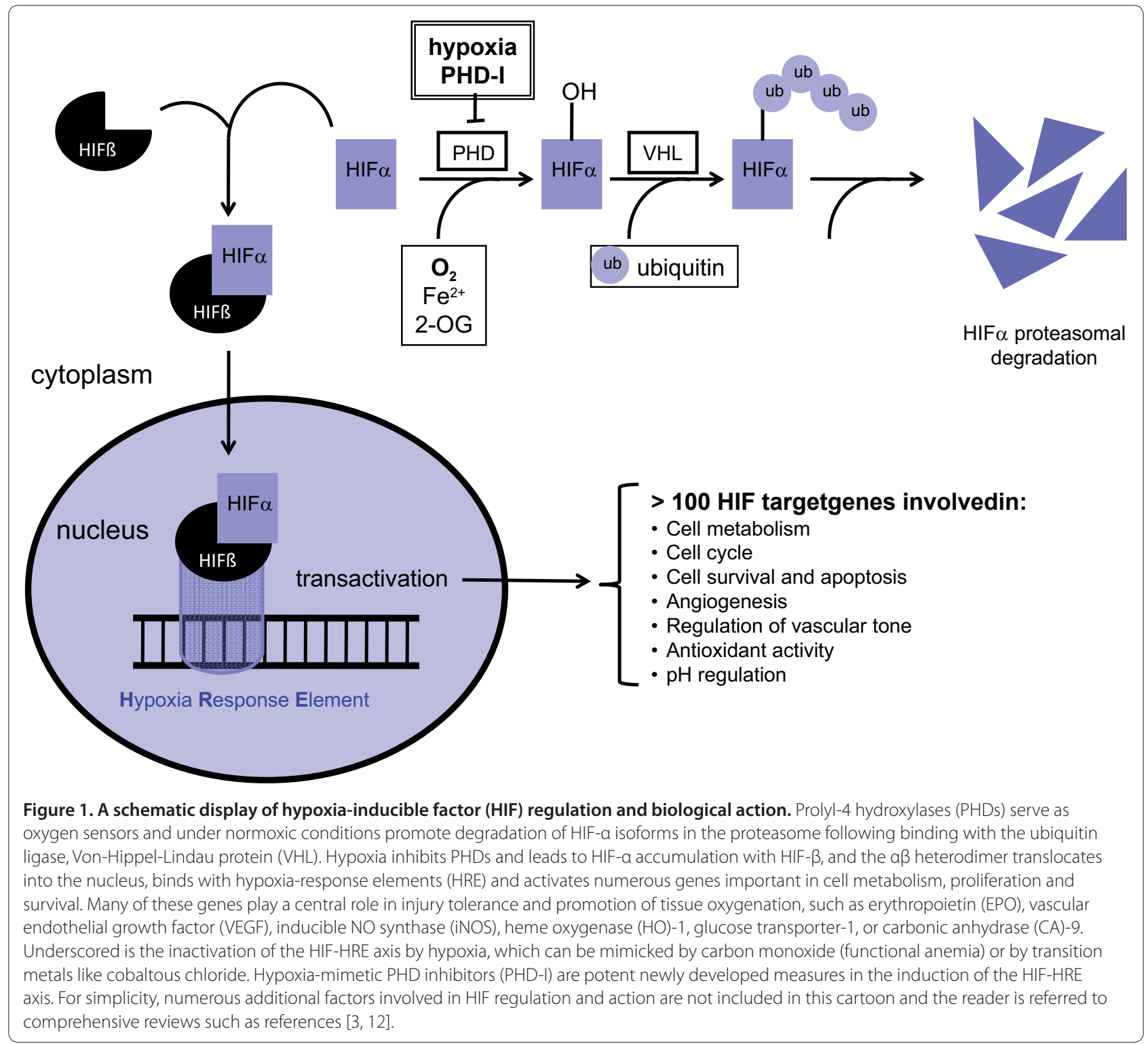

namely under cellular hypoxia. However, the threshold and extent of HIF activation may depend on the hypoxic stimulus and cell type involved. To some extent, these cellular variations may reflect different expression of various PHD isoforms in different tissues [5-7].

As HIF stimulation may potentiate hypoxia tolerance, studies were conducted to explore its clinical application. Widespread experimental hypoxic stimuli are listed in Table 1, all acting principally by the control of HIF- $\alpha$ degradation, initiated by PHDs. Except for carbon monoxide exposure, which is currently being tested in patients, none of these stimuli seems suitable for preconditional HIF activation in humans.

Apart from hypoxic stabilization, widely proven in vivo, HIF activation has also been demonstrated to occur under normal ambient oxygen tensions, mostly in cell cultures challenged with cytokines and growth factors. However, under stress, oxygen demand likely is increased, thus possibly leading to intracellular hypoxia even in cells kept under room air. For technical reasons, it is probably impossible to rule out such local cellular hypoxia that may exist predominantly within the mitochondria. Beyond this academic distinction between true cellular hypoxia and normoxia, it is important to recognize that clinical conditions, like inflammation, infection and sepsis, may lead to HIF activation. Thus, theoretically, cytokines or growth factors could be used for preconditional HIF activation in humans.

Although not a reasonable therapeutic intervention, strong and stable normoxic HIF activation can be 
Table 1. Modes of HIF signal enhancement

\begin{tabular}{|c|c|c|}
\hline Stimulus/Agent & Remarks & Potential Clinical Applications \\
\hline \multicolumn{3}{|c|}{ Inhibition of PHDs by the induction of cellular physiologic hypoxia } \\
\hline Hypoxic chamber (e.g., $8 \% \mathrm{O}_{2}$ in ambient air) & depressed systemic $\mathrm{PO}_{2}$ & \\
\hline Carbon monoxide admixture to ambient air & functional anemia normal systemic $\mathrm{PO}_{2}$ & $\checkmark$ \\
\hline Anemia & & normal systemic $\mathrm{PO}_{2}$ \\
\hline Arterial clamping & & normal systemic $\mathrm{PO}_{2}$ \\
\hline \multicolumn{3}{|l|}{ Chemical inhibition of PHDs by hypoxia-mimetics } \\
\hline $\mathrm{CoCl}_{2}$ (interferes with $\mathrm{Fe}^{2+}$ ) & non-specific & \\
\hline Mimosine (2-oxoglutarate analogue) & non-specific & \\
\hline Other patented PHD inhibitors & specific & $\checkmark$ \\
\hline \multicolumn{3}{|l|}{ Molecular biology techniques } \\
\hline Von-Hippel-Lindau knockout & non-specific & \\
\hline PHD siRNA transfection & PHD-specific & \\
\hline Constitutively active HIF-a transgenes & organ-specific & $\checkmark$ \\
\hline
\end{tabular}

PHD: prolyl hydroxylase domain enzyme

achieved by deletion of the VHL gene, which is a constant phenomenon in Von Hippel Lindau Disease and in renal clear cell carcinoma, and is also encountered in other tumors. Transgenic animals with VHL knockout serve to test the potential of HIF activation in ischemic/hypoxic diseases (C Rosenberger, unpublished data) [8]. Additional experimental probes for enhancing HIF signal are by transfection with PHD siRNA [9] or with the generation of constitutively active HIF- $\alpha$ transgenes [10].

So-called hypoxia mimetics block PHD activity, thus upregulating HIF under normoxia. PHDs require 2-oxoglutarate and ferrous iron as co-substrates. Nonspecific PHD inhibitors are either 2-oxoglutarate analogues or interfere with $\mathrm{Fe}^{2+}$. Recently, more specific PHD inhibitors (PHD) have been synthesized [11], and are currently being tested in animal and human studies.

Figure 1 represents a simplistic scheme of the canonical HIF regulation and action. Recent discoveries underscore a host of additional compound biological pathways, associated with the regulation of the HIF signal, including the control of HIF synthesis, HIF controlling PHD synthesis, putative competing/intervening impacts of HIF-3 $\alpha$ and PHD-3, cross-talk of HIF and other key regulators of gene expression (STAT, p-300 and others), further modification of HIF- $\alpha$ activity at the level of DNA hypoxiaresponsive elements by small ubiquitin-like modifiers (SUMO) and factor inhibiting HIF (FIH), and the effect of reactive oxygen species (ROS), NO and Krebs cycle metabolites on HIF degradation. These complex pathways are beyond the scope of this review, and the interested reader is referred to additional references [3,5,12-18].

\section{HIF expression under hypoxic stress and tissue injury}

The kidney serves as an excellent example for understanding HIF expression under hypoxic stress. Renal oxygenation is very heterogeneous, with $\mathrm{PO}_{2}$ falling to levels as low as $25 \mathrm{mmHg}$ in the outer medulla under normal physiologic conditions and to even lower values in the papilla $[3,4,19]$. Changes in renal parenchymal microcirculation and oxygenation have been thoroughly investigated in acute and chronic renal disorders $[19,20]$. Finally, the complex renal anatomy in which different cell types are in close proximity to regions with comparable ambient oxygenation, enables comparisons of cellular HIF response.

Interestingly, HIF expression is below detection threshold by immunostaining in the renal medulla, despite low physiologic ambient oxygenation (It should be emphasized that this statement regarding negative HIF immunostaining in the normally hypoxic medulla relates to kidneys perfusion-fixed in vivo without an interruption of renal oxygenation before fixation. Other modes of tissue harvesting for HIF determination, either by immunostaining or by molecular biology techniques may be falsely positive, as hypoxia-induced inhibition of PHD activity is instantaneous, and may lead to HIF- $\alpha$ stabilization even over short periods of hypoxia). Conceivably, this reflects the plasticity of HIF control to adjust for 'physiologically normal' oxygenation (i.e., adjusted rates of HIF- $\alpha$ generation and degradation under normal conditions.

Enhanced renal HIF- $\alpha$ is noted in rodents subjected to hypoxia or to inhaled carbon monoxide (chemical hypoxia) [21], and in hypoxic isolated perfused kidneys [22]. Different cells express diverse HIF isoforms: Whereas tubular segments express HIF- $1 \alpha$, HIF- $2 \alpha$ is principally produced by vascular endothelial and interstitial cells [21-23]. Interestingly, HIF-dependent genes are also selectively expressed in different cell types. For instance HIF-2-triggered EPO generation is specifically found in interstitial cells in the deep cortex [24]. In hypoxic 
isolated perfused kidneys, attenuation of severe medullary hypoxia by the inhibition of tubular transport markedly enhanced HIF expression, probably underscoring a window of opportunity to generate HIF and HIF-mediated adaptive responses only under moderate and sublethal hypoxic stress [22]. This pattern is consistent with HIF expression at the border of renal infarct zones only, indicating that dying cells within the critically ischemic region are incapable of mounting a hypoxia adaptive response [25].

We also found that HIF- $\alpha$ isoforms are stabilized in acute hypoxic stress, predominantly in the cortex in rhabdomyolysis-induced kidney injury [26], in the outer stripe of the outer medulla following ischemia and reperfusion $[27,28]$, or in the inner stripe and inner medulla following the induction of distal tubular hypoxic injury by radiocontrast agent, or after the inhibition of prostaglandin or NO synthesis or with their combinations [23]. Outer medullary HIF stabilization is also noted in chronic tubulointerstitial disease [29] and in experimental diabetes [30], again spatially distributed in areas with proven hypoxia. HIF was also detected in biopsies from transplanted kidneys [31]. Thus, HIF immunostaining is chronologically and spatially distributed in renal regions with abnormally low $\mathrm{PO}_{2}$.

Normal mice subjected to warm ischemia and reperfusion display limited injury only, as compared with extensive damage in HIF $(+/-)$ mice [32]. Thus, the importance of mounting an HIF response during hypoxic stress is undeniable.

Hypoxia-driven HIF stabilization during hypoxic stress has been encountered in other organs as well. HIF- $1 \alpha$ and PHD-2 expression increased in the neonatal rat brain following hypoxia [33] and HIF was detected in the hypoxic subendocardium [34] and in the ischemic liver [27]. HIF is also found within hypoxic regions in tumors, and may play an important role in tumor progression via upregulation of growth promoting and angiogenic factors [35].

\section{Potential usage of HIF modulation in clinical practice}

The impact of HIF stimulation on the expression of HIFdependent tissue-protective genes led to the expectation that timely upstream HIF stimulation may have great potential in the protection of endangered organs by downstream induction of protective genes [12]. Indeed, repeated systemic hypoxia, for instance, results in enhanced expression of renal HIF and HIF-dependent genes and attenuates warm-ischemic injury [36].

The use of hypoxia-mimetic PHD inhibitors is a promising potential new treatment option in diseases such as myocardial infarction, stroke, renal or liver injury, peripheral vascular disease, or severe anemia. Studies with PHD inhibitors and other manipulations of HIF upregulation favor this hypothesis [11].

\section{Anemia}

Specific PHD inhibitors induce HIF- $2 \alpha$ expression in interstitial fibroblasts in the deep cortex [24], enhance erythropoietin generation, and were found to provoke erythrocytosis in primates [37]. Phase 2 clinical trials in patients with chronic kidney disease are currently under way, studying the effect of oral PHD inhibitors as potential substitutes to EPO injection.

\section{Acute kidney injury}

The potential protective impact of HIF upregulation by PHD inhibitors has been extensively studied in acute kidney injury. In isolated kidneys perfused with lowoxygen containing medium, pre-treatment with a PHD inhibitor improved renal blood flow and attenuated medullary hypoxic damage [38]. Conditional inactivation of VHL in mice (hence HIF stabilization) resulted in tolerance to renal ischemia and reperfusion [8] and to rhabdomyolysis-induced acute kidney injury (Rosenberger $C$, unpublished data). Whereas gene transfer of negative-dominant HIF led to severe damage in the normally hypoxic renal medulla in intact rats, transfer of constitutively active HIF (HIF/VP16) induced expression of various HIF-regulated genes and protected the medulla against acute ischemic insults [39]. Furthermore, in rats and mice subjected to warm ischemia and reflow, PHD inhibitors and carbon monoxide pre-treatment (i.e., functional anemia) markedly attenuated kidney damage and dysfunction [32,40]. Donor pre-treatment with a PHD inhibitor also prevented graft injury and prolonged survival in an allogenic kidney transplant model in rats [41]. Finally, rats preconditioned by carbon monoxide, displayed reduced cisplatin renal toxicity, with attenuation of renal dysfunction and the extent of tubular apoptosis and necrosis [42]. Taken together, all these observations indicate that HIF stabilization seemingly is a promising novel interventional strategy in acute kidney injuries [12].

\section{Myocardial injury}

Activation of the HIF system has also been found to be cardioprotective. In a model of myocardial ischemia in rabbits, pre-treatment with a PHD inhibitor induced robust expression of HO-1 and markedly attenuated infarct size and myocardial inflammation [43]. In another report, PHD inhibitors did not reduce infarct size, but improved left ventricular function and prevented remodeling [44]. In the same fashion, selective silencing of PHD-2 with siRNA $24 \mathrm{~h}$ before global myocardial ischemia/reperfusion in mice reduced the infarct size by $70 \%$ and markedly improved left ventricular systolic 
function [9]. Remote preconditioning by intermittent renal artery occlusion also resulted in cardiac protection, conceivably through PHD inhibition [45].

Enhanced levels of PHD-3 were traced in the hibernating myocardium [34] and in end-stage heart failure in humans, associated also with elevated HIF- $3 \alpha$ [46] (which may act as a competitive inhibitor of active HIF- $\alpha$ isoforms [14]). Thus, PHD inhibitors may conceivably also be beneficial in these disorders. Finally, cardioprotection during heat acclimation is also mediated in part by HIF upregulation [47], providing another potential situation for the administration of PHD inhibitors.

\section{Neuronal injuries}

The effect of PHD inhibitors has also been assessed in disorders of the central nervous system. In vitro, rotenoneinduced neuronal apoptosis was attenuated and autophagy increased, as the result of enhanced HIF following deferoxamine administration [48]. In vivo, PHD inhibitors have shown promising results in the attenuation of ischemic stroke [49], and might be neuroprotective in metabolic chronic neurodegenerative conditions [50]. However, studies showing inhibition of PHD-1 by ROS suggest non-HIF-mediated neuronal protection under normoxic conditions [51].

\section{Lung injury}

Preterm lambs developing respiratory distress syndrome display upregulation of PHDs with a reciprocal fall in HIF- $\alpha$ isoforms and HIF-dependent VEGF [53]. This observation implies that PHD inhibitors might have therapeutic potential in this clinical setup.

\section{Liver disease}

Hepatic HIF-1 $\alpha$ is upregulated following warm ischemia [27], and is required for restoration of gluconeogenesis in the regenerating liver [52], implying yet another potential use for PHD inhibitors in acute liver disease.

\section{Peripheral vascular disease}

In a model of limb ischemia in mice, PHD inhibitors enhanced HIF expression and downstream VEGF and VEGF-receptor Flk-1, leading to improved capillary density, indicating a potential therapeutic use of PHD inhibitors in promoting angiogenesis in ischemic diseases, such as severe peripheral vascular disease [54]. Transfection with HIF- $1 \alpha$, combined with PHD inhibitortreated bone marrow-derived angiogenic cells increased perfusion, motor function, and limb salvage in old mice with ischemic hind limbs [55]. Results of a phase-1 study in patients with critical limb ischemia indicate that transfection with a constitutively active form of HIF- $1 \alpha$ might also promote limb salvage [10]. Further clinical trials with PHD inhibitors are currently under way in burn wound healing and salvage of critically ischemic limbs.

\section{Oxidative stress}

Enhanced cellular ROS concentrations, as happens with shock and tissue hypoxia, result in increased PHD activity, and this effect is antagonized by ROS scavengers [15]. This situation may lead to HIF de-stabilization and inadequate HIF response to hypoxia. For example, hypoxia-mediated HIF expression in the diabetic renal medulla is substantially improved by the administration of the membrane-permeable superoxide dismutase mimetic tempol [30]. It is, therefore, tempting to assume that ROS scavengers, as well as PHD inhibitors may improve tissue adaptive responses to hypoxia, coupled with oxidative stress. However, contradicting evidence exists, indicating that ROS might trigger HIF in the absence of hypoxia. This has been suggested by studying liver tissue in acetaminophen-induced liver injury, before the development of overt liver injury and hypoxia [56], and in aged well-fed animals [57]. The role of HIF stimulation during oxidative stress therefore needs further assessment.

\section{Important considerations}

HIF stimulation is not all-protective. The wide range of HIF-dependent genes, and its tight cross-communication with other key regulators of gene expression [13,58,59] raise concern regarding concomitant non-selective activation of protective as well as harmful systems. Among potential unwanted outcomes is the enhancement of tumor growth [60], promotion of fibrosis [61] or the induction of pre-eclampsia in pregnant women [62]. Indeed, whereas HIF activation is considered renoprotective in acute kidney injury, it may play a role in the progression of chronic kidney disease and certainly is an important factor in the promotion of renal malignancy [3,20].

Diverse characteristics and distribution patterns of different PHDs [5-7] and particular actions of various PHD inhibitors [11,37] might enable selective manipulation of the HIF system in a more desired way, selectively favoring advantageous HIF-dependent responses in preferred tissues. Furthermore, it is believed that activation of adverse responses requires protracted HIF stimulation, whereas short-term and transient HIF activation might suffice to activate tissue-protective systems without continuing induction of harmful systems. However, this concept needs confirmation in clinical trials.

\section{Conclusion}

Elucidating the mechanisms involved in HIF-mediated cellular responses to acute hypoxic stress has led to the 
discovery of novel potential therapeutic options for the prevention or attenuation of tissue injury. The non-selective enhancement of gene expression by current modes of HIF augmentation warrants caution, since undesired enhancement of certain genes may be hazardous.

We anticipate that in the coming years the use of PHD inhibitors and other stimulants of the HIF system will be tested in many clinical scenarios associated with critical care and emergency medicine, while HIF silencing strategies may be tested in chronic diseases, such as malignancies and disorders with enhanced tissue scarring.

\section{Acknowledgement}

This report was supported by the Israel Science Foundation (Grant No.

1473/08) and the Harvard Medical Faculty Physicians at Beth Israel Deaconess

Medical Center, Boston, MA.

\section{Competing interests}

The authors declare that they have no competing interests.

\section{List of abbreviations used}

EPO: erythropoietin; FIH: factor inhibiting HIF; HIF: hypoxia-inducible factors; HO: heme-oxygenase; HRE: hypoxia response elements; HSP: heat-shock proteins; PHD: prolyl-4-hyrdoxylase domain enzymes; ROS: reactive oxygen species; SUMO: small ubiquitin-like modifiers; VEGF: vascular endothelial growth factor; VHL:Von-Hippel-Lindau protein.

\section{Author details}

'Department of Medicine, Hadassah Hosptial, Mt. Scopus, PO Box 24035, 91240 Jerusalem, Israel. '2Department of Pathology, Beth Israel Deaconess Medical Center and Harvard University, 330 Brookline Avenue, Boston, MA 02215, USA. ${ }^{3}$ Department of Trauma/General Surgery, UPMC - Presbyterian Hospital, F1266 Lothrop Street, Pittsburgh, PA, 15213, USA.

Published: 22 March 201

\section{References}

1. Bernhardt WM, Eckardt KU: Physiological basis for the use of erythropoietin in critically ill patients at risk for acute kidney injury. Curr Opin Crit Care 2008, 14:621-626.

2. Semenza G: Signal transduction to hypoxia-inducible factor 1. Biochem Pharmacol 2002, 64:993-998.

3. Gunaratnam L, Bonventre JV: HIF in kidney disease and development. J Am Soc Nephrol 2009, 20:1877-1887.

4. Rosenberger C, Rosen S, Heyman S: Current understanding of HIF in renal disease. Kidney Blood Press Res 2005, 28:325-340.

5. Willam C, Maxwell PH, Nichols L, et al: HIF prolyl hydroxylases in the rat; organ distribution and changes in expression following hypoxia and coronary artery ligation. J Mol Cell Cardiol 2006, 41:68-77.

6. Li N, Yi F, Sundy CM, et al:: Expression and actions of HIF prolyl-4hydroxylase in the rat kidneys. Am J Physiol Renal Physiol 2007. 292:F207-F216

7. Schodel J, Klanke B, Weidemann A, et al.: HIF-prolyl hydroxylases in the rat kidney: physiologic expression patterns and regulation in acute kidney injury. Am J Pathol 2009, 174:1663-1674.

8. Iguchi M, Kakinuma Y, Kurabayashi A, et al:: Acute inactivation of the VHL gene contributes to protective effects of ischemic preconditioning in the mouse kidney. Nephron Exp Nephrol 2008, 110:e82-e90.

9. Natarajan R, Salloum FN, Fisher BJ, Kukreja RC, Fowler III AA: HIF-1 activation by prolyl 4-hyroxylase-2 gene silencing attenuates myocardial ischemiareperfusion injury. Circ Res 2006, 98:133-140.

10. Rajagopalan S, Olin J, Deitcher S, et al:: Use of a constitutively active hypoxia-inducible factor-1alpha transgene as a therapeutic strategy in no-option critical limb ischemia patients: phase I dose-escalation experience. Circulation 2007, 115:1234-1243.

11. Myllyharju J: HIF prolyl 4-hydroxylases and their potential as drug targets. Curr Pharm Des 2009, 15: 3878-3885.
12. Nangaku M: Novel therapeutic approach targeting the HIF-HRE system in the kidney. Adv Exp Med Biol 2009, 645:81-86.

13. Webb JD, Coleman ML, Pugh CW: Hypoxia, hypoxia-inducible factors (HIF), HIF hydroxylases and oxygen sensing. Cell Mol Life Sci 2009, 66:3539-3554.

14. Tanaka T, Wiesener M, Bernhardt W, Eckardt KU, Warnecke C: The human HIF (hypoxia-inducible factor)-3alpha gene is a HIF-1 target gene and may modulate hypoxic gene induction. Biochem J 2009, 424:143-151

15. Callapina M, Zhou J, Schmid T, Kohl R, Brune B: NO restores HIF-1 alpha hydroxylation during hypoxia: role of reactive oxygen species. Free Radic Biol Med 2005, 39:925-936

16. Haase VH: The VHL/HIF oxygen-sensing pathway and its relevance to kidney disease. Kidney Int 2006, 69:1302-1307.

17. Koivunen P, Hirsila M, Remes AM, Hassinen IE, Kivirikko Kl, Myllyharju J: Inhibition of hypoxia-inducible factor (HIF) hydroxylases by citric acid cycle intermediates: possible links between cell metabolism and stabilization of HIF. J Biol Chem 2007, 282:4524-4532.

18. Serra-Perez A, Planas AM, Nunez-O'Mara A, et al.: Extended ischemia prevents HIF1alpha degradation at reoxygenation by impairing prolylhydroxylation: role of Krebs cycle metabolites. J Biol Chem 2010 285:18217-1824

19. Rosenberger C, Rosen S, Heyman SN: Renal Parenchymal Oxygenation and Hypoxia Adaptation in Acute Kidney Injury. Clin Exp Pharmacol Physiol 2006 33:980-988

20. Heyman SN, Khamaisi M, Rosen S, Rosenberger C: Renal parenchymal hypoxia, hypoxia response and the progression of chronic kidney disease. Am J Nephrol 2008, 28:998-1006.

21. Rosenberger C, Mandriota S, Jurgensen JS, et al: Expression of hypoxiainducible factor-1alpha and -2alpha in hypoxic and ischemic rat kidneys. J Am Soc Nephrol 2002, 13:1721-1732.

22. Rosenberger C, Shina A, Rosen S, Goldfarb M, Eckardt K, Heyman SN: Hypoxia inducible factors and tubular cell survival in isolated perfused kidneys. Kidney Int 2006, 70:60-70.

23. Rosenberger C, Heyman SN, Rosen S, et al.: Upregulation of HIF in acute renal failure - evidence for a protective transcriptional response to hypoxia. Kidney Int 2005, 67:531-542

24. Paliege A, Rosenberger C, Bondke A, et al:. Hypoxia-inducible factor-2aexpressing interstitial fibroblasts are the only renal cells that express erythropoietin under hypoxia-inducible factor stabilization. Kidney Int 2010, 77:312-318.

25. Rosenberger C, Griethe W, Gruber G, et al: Cellular responses to hypoxia after renal segmental infarction. Kidney Int 2003, 64:874-886.

26. Rosenberger C, Goldfarb M, Shina A, et al: Evidence for sustained renal hypoxia and transient hypoxia adaptation in experimental rhabdomyolysis-induced acute kidney injury. Nephrol Dial Transp/ 2008, 23:1135-1143

27. Cursio R, Miele C, Filippa N, Van Obberghen E, Gugenheim J: Liver HIF-1 alpha induction precedes apoptosis following normothermic ischemiareperfusion in rats. Transplant Proc 2008, 40:2042-2045.

28. Heyman SN, Rosenberger C, Rosen S: Experimental ischemia-reperfusion - biases and myths: the proximal vs. distal hypoxic tubular injury debate revisited. Kidney Int 2010, 77:9-16.

29. Goldfarb M, Rosenberger C, Abassi Z, et al:: Acute-on-chronic renal failure in the rat: Functional compensation and hypoxia tolerance. Am J Nephrol 2006, 26:22-33.

30. Rosenberger $\mathrm{C}$, Khamaisi $\mathrm{M}$, Abassi Z, et al:: Adaptation to hypoxia in the diabetic rat kidney. Kidney Int 2008, 73:34-42.

31. Rosenberger C, Pratschke J, Rudolph B, et al:: Immunohistochemical Detection of Hypoxia-Inducible Factor-1alpha in Human Renal Allograft Biopsies. J Am Soc Nephrol 2007, 18:343-351.

32. Hill P, Shukla D, Tran MG, et al:: Inhibition of hypoxia inducible factor hydroxylases protects against renal ischemia-reperfusion injury. $J$ Am SoC Nephrol 2008, 19:39-46.

33. Jones NM, Lee EM, Brown TG, Jarrott B, Beart PM: Hypoxic preconditioning produces differential expression of hypoxia-inducible factor-1alpha (HIF-1alpha) and its regulatory enzyme HIF prolyl hydroxylase 2 in neonatal rat brain. Neurosci Lett 2006, 404:72-77.

34. May D, Gilon D, Djonov V, et al:: Transgenic system for conditional induction and rescue of chronic myocardial hibernation provides insights into genomic programs of hibernation. Proc Natl Acad Sci USA 2008, 105:282-287.

35. Pugh CW, Ratcliffe PJ: Regulation of angiogenesis by hypoxia: role of the 
HIF system. NatMed 2003, 9:677-684.

36. Yang CC, Lin LC, Wu MS, Chien CT, Lai MK: Repetitive hypoxic preconditioning attenuates renal ischemia/reperfusion induced oxidative injury via upregulating HIF-1 alpha-dependent bcl-2 signaling. Transplantation 2009, 88:1251-1260.

37. Hsieh MM, Linde NS, Wynter A, et al.: HIF prolyl hydroxylase inhibition results in endogenous erythropoietin induction, erythrocytosis, and modest fetal hemoglobin expression in rhesus macaques. Blood 2007 110:2140-2147.

38. Rosenberger C, Rosen S, Shina A, et al:: Activation of hypoxia inducible factors (HIF) ameliorates hypoxic distal tubular injury in the isolated perfused rat kidney. Nephrol Dial transplant 2008, 23:3472-3478

39. Manotham K, Tanaka T, Ohse T, et al:: A biologic role of HIF-1 in the renal medulla. Kidney Int 2005, 67:1428-1439.

40. Bernhardt WM, Campean V, Kany S, et al:: Preconditional activation of hypoxia-inducible factors ameliorates ischemic acute renal failure. J Am Soc Nephrol 2006, 17:1970-1978.

41. Bernhardt WM, Gottmann U, Doyon F, et al:: Donor treatment with a PHDinhibitor activating HIFs prevents graft injury and prolongs survival in an allogenic kidney transplant model. Proc Natl Acad Sci USA 2009, 106:21276-21281.

42. Weidemann A, Bernhardt WM, Klanke B, et al.: HIF activation protects from acute kidney injury. J Am Soc Nephrol 2008, 19:486-494.

43. Ockaili R, Natarajan R, Salloum F, et al: HIF-1 activation attenuates postischemic myocardial injury: role for heme oxygenase-1 in modulating microvascular chemokine generation. Am J Physiol Heart Circ Physiol 2005, 289: $\mathrm{H} 542-\mathrm{H} 548$

44. Philipp S, Jurgensen JS, Fielitz J, et al: Stabilization of hypoxia inducible factor rather than modulation of collagen metabolism improves cardiac function after acute myocardial infarction in rats. Eur J Heart Fail 2006 8:347-354.

45. Kant R, Diwan V, Jaggi AS, Singh N, Singh D: Remote renal preconditioning induced cardioprotection: a key role of hypoxia inducible factor-prolyl 4-hydroxylases. Mol Cell Biochem 2008, 312:25-31.

46. Zolk O, Solbach TF, Eschenhagen T, Weidemann A, Fromm MF: Activation of negative regulators of the hypoxia-inducible factor (HIF) pathway in human end-stage heart failure. Biochem Biophys Res Commun 2008, 376:315-320

47. Horowitz M, Assadi H: Heat acclimation-mediated cross-tolerance in cardioprotection: do HSP70 and HIF-1alpha play a role? Ann N Y Acad Sci 2010, 1188:199-206

48. Wu Y, LiX Xie W, Jankovic J, Le W, Pan T. Neuroprotection of deferoxamine on rotenone-induced injury via accumulation of HIF-1 alpha and induction of autophagy in SH-SY5Y cells. Neurochem Int 2010, 57: 98-205.

49. Harten SK, Ashcroft M, Maxwell PH: Prolyl hydroxylase domain inhibitors: a route to HIF activation and neuroprotection. Antioxid Redox Signal 2010 $12: 459-480$
50. Niatsetskaya Z, Basso M, Speer RE, et al.: HIF prolyl hydroxylase inhibitors prevent neuronal death induced by mitochondrial toxins: therapeutic implications for Huntington's disease and Alzheimer's disease. Antioxid Redox Signal 2010, 12:435-443.

51. Siddiq A, Aminova LR, Troy CM, et al: Selective inhibition of hypoxiainducible factor (HIF) prolyl-hydroxylase 1 mediates neuroprotection against normoxic oxidative death via HIF- and CREB-independent pathways. J Neurosci 2009, 29:8828-8838

52. Grover TR, Asikainen TM, Kinsella JP, Abman SH, White CW: Hypoxia-inducible factors HIF-1alpha and HIF-2alpha are decreased in an experimental model of severe respiratory distress syndrome in preterm lambs. Am J Physiol Lung Cell Mol Physio/ 2007, 292:L1345-L1351.

53. Tajima T, Goda N, Fujiki N, et al:: HIF-1alpha is necessary to support gluconeogenesis during liver regeneration. Biochem Biophys Res Commun 2009, 387:789-794

54. Milkiewicz M, Pugh CW, Egginton S: Inhibition of endogenous HIF inactivation induces angiogenesis in ischaemic skeletal muscles of mice. J Physiol 2004, 560:21-26.

55. Rey S, Lee K, Wang CJ, et al.: Synergistic effect of HIF-1alpha gene therapy and HIF-1-activated bone marrow-derived angiogenic cells in a mouse model of limb ischemia. Proc Natl Acad Sci USA 2009, 106: 20399-20404.

56. James LP, Donahower B, Burke AS, McCullough S, Hinson JA: Induction of the nuclear factor HIF-1alpha in acetaminophen toxicity: evidence for oxidative stress. Biochem Biophys Res Commun 2006, 343:171-176.

57. Kang MJ, Kim HJ, Kim HK, et al.: The effect of age and calorie restriction on HIF-1-responsive genes in aged liver. Biogerontology 2005, 6:27-37.

58. Serchov T, Dubois-Pot-Schneider H, Charlot C, Rosl F, Wasylyk B: Involvement of net and HIF-1alpha in distinct yet intricately linked hypoxia-induced signaling pathways. J Bio/ Chem 2010, 285:21223-21232

59. Nechemia-Arbely Y, Rosenberger C, Khamaisi M, Koesters R, Shina A, Klaus S, Shriki A, Rosen S, Axelrod JH, Heyman SN: Renal hypoxia inducible factors (HIF) and STAT3 cross-talk in vivo [abstract]. J Am Soc Nephrol 2009 20:F-FC184

60. Jokilehto T, Jaakkola PM: The role of HIF prolyl hydroxylases in tumour growth. J Cell Mol Med 2010, 14:.758-770

61. Higgins DF, Kimura K, Bernhardt WM, et al:: Hypoxia promotes fibrogenesis in vivo via HIF-1 stimulation of epithelial-to-mesenchymal transition. J Clin Invest 2007, 117: 3810-3820

62. Nevo O, Soleymanlou N, Wu Y, et al:: Increased expression of sFlt-1 in in vivo and in vitro models of human placental hypoxia is mediated by HIF-1. Am J Physiol Regul Integr Comp Physiol 2006, 291:R1085-R1093.

doi:10.1186/cc9991

Cite this article as: Heyman SN, et al:: Hypoxia-inducible factors and the prevention of acute organ injury. Critical Care 2011, 15:209. 